\title{
MORPHOLOGICAL AND CULTURAL VARIABILITY OF fusarium solani ISOLATES FROM CHILLI (capsicum annuum L)
}

\author{
M. J. Patil ${ }^{1}$, Ashish Lambat ${ }^{2}$, Rajesh Gadewar ${ }^{2}$ and Sanjeev Charjan' \\ 1 Dr. PDKV's College of Agriculture, Nagpur M.S. India \\ 2 Sevadal Mahila Mahavidyalaya and Research Academy, Nagpur, M.S. \\ India Corresponding Author: lambatashish@gmail.com

\section{Abstract:} \\ Considerable morphological and cultural variability existed among the isolates of Fusarium \\ solani from chilli (Capsicum annuum L). The isolates showed slow to rapid growth and variable \\ pigmentation. On the basis of the radial growth on potato dextrose medium in $\mathrm{mm}$, these \\ isolates were categorized into five groups such as Nine isolates very slow $(<50 \mathrm{~mm})$, Seven \\ isolates Slow growth (51-60 mm), Eight isolates medium growth (61-70), 17 isolates showed \\ fast growth and fifteen isolates possessing more than $80 \mathrm{~mm}$ and above radial growth hence \\ categorized under very fast growing group.
}

\section{Key words:}

Fusarium solani. Chilli, Variability

\section{Introduction:}

Although the chilli crop is susceptible to Damping off, dieback, fruit rot, and powdery mildew but wilt seems to be a new dimension for studying the various aspects. The incidence of wilt is gradually increasing particularly in black cotton soil of irrigated tract. Not much work has been done in respect of chilli caused by Fusarium solani. Recently it appears to be major pathogen causing losses nearly 5 to 100 per cent. The production and productivity of chilli are constrained due to the incidence of Fusarial wilt, a soil borne disease that causes yield loss to the tune of 50-80 per cent. (Madhavi, et al, 2006) the spread of disease is very fast particularly in chilli growing areas of

Maharashtra. The disease can be located mainly in vertisols having over irrigation and ill drained soils. The disease can be seen in all high yielding varieties of chilli hence there is need to understand the diversity of Fusarium isolates collected from different geographical area of Vidarbha, Maharashtra. 


\section{Materials And Method:}

Survey was conducted in different areas of Vidarbha region of Mahaharashtra. On Fusarium wilt of chilli during crop season 2007-2008. The roots of completely and partially wilted plants of chilli (Capsicum anmmm L) suspected to be infected with Fusarium solani were collected from the field in which most plant exhibited disease symptoms. Pure cultures of the isolates of pathogen from the diseased plants were established by using single hyphal tip technique and pure culture was obtained. The representative cultures were sent to Indian type culture collection ((ITCC) 1AR1, New Delhi for identification. The cultures were identified using keys (Booth, 1971) and compared with reference cultures identified at IARI, New Delhi as $F$. solani. The remaining cultures were microscopically examined and identified as $F$. solani. On the basis of morphological characters of the fungus, forty eight pure cultures collected from different regions and four cultures received from department of Plant Pathology college of Raichur, Karnataka were maintained on potato dextrose medium slants for further study.

All the 52 isolates of $F$. solani were characterized on the basis of their cultural characteristics on PDA. The type of mycelia colony growth pattern and pigmentation was recorded on $7^{\text {th }}$ days of inoculation of pathogen on PDA medium. Produced in substrate was observed during the incubation period at $27 \pm 1^{0} \mathrm{C}$.

\section{Results And Discussion:}

All the 52 isolates of $F$. solani were characterized on the basis of their cultural characteristic on PDA (Table-1). The isolates collected were designated as FS-1 to FS-52. The general variation in cultural characteristics was observed among the isolates of $F$. solani. Mycelial growth of isolates varied in the form of dull white, submerged, cottony white, submerged to raised, profuse fluffy white, fussy cottony developed in petriplates and reddish or brownish colour developed at backside of the petriplates (FS-15) Fussy raised star like 
appearance in (FS-14) white submerged on petriplates and reddish colour on the backside of the petriplates in (FS-25) yellowish white but greenish colour at the centre (FS-20) indicates the wide variation among isolates of $F$. solani. Maximum growth $(89.44 \mathrm{~mm})$ was observed in FS-11 followed by FS-9 and FS13 recording 87.50 and $87.33 \mathrm{~mm}$ respectively. All these three isolates were obtained from Amravati district. The lowest growth was observed in FS-1 and FS-6 (41.66 mm each) followed by FS-41 and FS-8 exhibiting 44.33 and 45.66 $\mathrm{mm}$ respectively. These isolates were obtained from Akola and FS-41 from Bhandara district. The pigmentation also reflects the variation among isolates of $F$. solani. Three milky white, 8 dull white to white, 4 reddish brown, 7 yellowish, 3 purple, 11 light red and 10 brownish to dark brown pigmentation was noticed in different isolates. Different workers have also reported the differences in cultural characters of pathogens belonging to same genus and species. The findings of the present results were also on the line of work reported by Patil et al. (2005), Randhawa et.al. (2006) Patil et al. (2008), Murumkar and Deshpande (2009), Patel et al. (2011) and Singh et al. (2011). On the basis of radial mycelial growth on potato dextrose agar medium, 52 isolates of F. solani causing chilli wilt were categorized. (Table-2). Five isolates viz.FS-1, FS-6, FS-8, FS-41 and FS-28 had shown very slow growth rate after 7 days of incubation at $27 \pm 1^{\circ} \mathrm{C}(<50 \mathrm{~mm})$, Slow growth $(51-60 \mathrm{~mm})$ was noticed in seven isolates, Eight isolates showing a range of 61 to $70 \mathrm{~mm}$ radial growth were categorized as medium growth. Seventeen isolates were categorized as fast growth which ranged between $71-80 \mathrm{~mm}$ growths. Another very fast rate group encompasses fifteen isolates possessing more than $80 \mathrm{~mm}$ and above radial mycelial growth. Fifteen different isolates has categorized in this group. Earlier workers also grouped the isolates on the basis of growth rates while working with different fungus causing diseases. Similar types of grouping were made in the present investigations as adopted by Paulikar and Raut (2004), Giri (2002), Honnareddy and Dubey (2007), Patil Kulkarni et al. (2008) and Murumkar and Deshpande (2009) for Fusarium oxysporum F. sp. ciceri, F. 
udum, F. oxysporum F. sp. ciceri, F. oxysporum F. sp. gladioli and F. oxysporum F. sp. earthemi respectively.

\section{Conclusion:}

Fusarium wilt isolates were collected from varied places and hence reflect the changes in morphological and cultural characters. The present findings revealed high level of morphological and cultural diversities among the isolates of Fusarium solani collected from various locations of Vidarbha region of Maharashtra.

\section{References:}

Booth, C. 1971. The Genus Fusarium. Common Wealth Mycological Institute, New England.

Giri, G. K. 2002. Pathogenic variability in Fusarium udum Butler. Ph.D. Thesis (Unpub.) Submitted to Dr. PDKV, Akola.

Honnareddy, N. and S.C. Dubey. 2007. Morphological characterization of Indian isolates of Fusarium oxysporum F. sp. ciceri causing chickpea wilt. Indian Phytopath; 60(3): 373-376.

Madhavi, M. C. Pramod Chandra Kumar, D. Raja Ram Reddy and TVK Singh. 2006. Integrated management of wilt of chilli incited by Fusarium solani. Indian J. PI. Protec; 34(2): 225-228.

Murumkar, D.R. and A.N. Deshpande. 2009. Variability among isolates of Fusarium oxysporum F. sp. carthami. J. Maharashtra Agric. Univ; 34(2): 178-180.

Patel, S. I., R. L. Patel., A. G. Desai and D. S. Patel. 2011. Morphological, cultural and Pathogenic variability among Fusarium udum and root dip 
inoculation technique for screening Pigeon pea germplasm. J. Mycol. PI.

Pathol; 41(1): 57-62.

Patil Kulkarni, Sumitra, Yashoda R. Hegde and Shrikant Kulkarni. 2008. Molecular and morphological variability among the isolates of Fusarium oxysporum F. sp. gladioli causing wilt of gladiolus. Indian J. Crop Science; 3(1):155-157.

Patil, Pushpa D., S.G. Borkar and G.N. Dake. 2006. Use of culture filtrate of Fusarium oxysporum F. sp. ciceri to identify resistance chickpea callus to wilt. J. Mycol. PI. Pathol; 36(2): 185-188.

Paulkar, P. K. and B. T. Raut. 2004. Variability among isolates of Fusarium oxysporum F. sp. ciceri. J. Mycology and PI. Pathol; 34(1): 20-23.

Randhawa, Mandeep., K.S. Sandhu and Singh Pushpinder Paul. 2006. Morphological characterization and pathogenic ability of causal organism of muskmelon wilt. PI. Dis. Res; 21 (1):55-57.

Singh, S. K., Bikram Singh., V. B. Singh and Reena. 2011. Morphological, cultural and pathogenic variability among the isolates of Fusarium oxysporum F. sp. ciceri causing wilt of chickpea. Ann. PI. Protec. Sci; 19(1): 155-158. 
Table-1: Growth of various Fusarium solani isolates on Potato dextrose agar medium

\begin{tabular}{|c|c|c|c|c|}
\hline $\begin{array}{l}\text { Sr. } \\
\text { No. }\end{array}$ & $\begin{array}{l}\text { Desig- } \\
\text { nated as }\end{array}$ & Colony characters & $\begin{array}{c}\begin{array}{c}\text { Colony } \\
\text { diameter } \\
(\mathbf{m m})\end{array} \\
\text { Mean }\end{array}$ & Pigmentation \\
\hline 1 & FS-1 & Dull white submersed growth & 41.66 & Milky white \\
\hline 2 & FS-2 & $\begin{array}{l}\text { Mycellium cottony white } \\
\text { suppressed aerial at centre }\end{array}$ & 53.33 & Light Red \\
\hline 3 & FS-3 & $\begin{array}{l}\text { Submerged white slow to medium } \\
\text { growth }\end{array}$ & 62.11 & Purple \\
\hline 4 & FS-4 & Dull white submerged growth & 59.33 & Purple \\
\hline 5 & FS-5 & Profuse fluffy white & 76.22 & Purple \\
\hline 6 & FS-6 & Aerial loose purple white growth & 41.66 & Dull white \\
\hline 7 & FS-7 & $\begin{array}{l}\text { Yellow to white fussy cottony raised } \\
\text { growth }\end{array}$ & 68.33 & Light Red \\
\hline 8 & FS-8 & $\begin{array}{l}\text { Cottony mycelium, submerged fast } \\
\text { growth }\end{array}$ & 45.66 & Dull white \\
\hline 9 & FS-9 & White fussy cottony growth & 87.50 & Light Yellow \\
\hline 10 & FS-10 & Dull white submerged growth & 72.44 & - \\
\hline 11 & FS-11 & Brick red submerged growth & 89.44 & Yellowish \\
\hline 12 & FS-12 & White yellowish raised growth & 83.66 & Milky white \\
\hline 13 & FS-13 & Dull white submerged growth & 87.33 & Brownish \\
\hline 14 & FS-14 & Fussy raised star like appearance & 78.50 & Dark Brown \\
\hline 15 & FS-15 & $\begin{array}{l}\text { Cottony fussy raised growth, } \\
\text { reddish colour developed at the } \\
\text { back side of petriplates }\end{array}$ & 65.00 & Light red \\
\hline 16 & FS-16 & $\begin{array}{l}\text { Cottony white medium to fast } \\
\text { submersed growth }\end{array}$ & 65.33 & Light red \\
\hline 17 & FS-17 & $\begin{array}{l}\text { White sraw colour. dusty black at } \\
\text { centre and brown around it }\end{array}$ & 84.66 & Light red \\
\hline 18 & FS-18 & $\begin{array}{l}\text { Dull white to yellowish suppressed } \\
\text { growth }\end{array}$ & 81.33 & Yellowish \\
\hline 19 & FS-19 & White creamy raised growth & 71.66 & $\begin{array}{l}\text { Reddish } \\
\text { brown }\end{array}$ \\
\hline 20 & FS-20 & $\begin{array}{l}\text { Yellowish white but greenish colour } \\
\text { at centre submerged growth }\end{array}$ & 74.44 & Whitish \\
\hline 21 & FS-21 & Dull white yellowish raised growth & 81.11 & - \\
\hline 22 & FS-22 & $\begin{array}{l}\text { Cottony fussy raised growth } \\
\text {.reddish brown colour developed at } \\
\text { back side of petriplates }\end{array}$ & 78.33 & Dark brown \\
\hline 23 & FS-23 & Cottony white submerged growth & 68.33 & $\begin{array}{l}\text { Reddish } \\
\text { brown }\end{array}$ \\
\hline 24 & FS-24 & $\begin{array}{l}\text { Medium dull white cottony raised } \\
\text { growth }\end{array}$ & 71.55 & $\begin{array}{l}\text { Reddish } \\
\text { brown }\end{array}$ \\
\hline
\end{tabular}




\begin{tabular}{|c|c|c|c|c|}
\hline $\begin{array}{l}\text { Sr. } \\
\text { No. }\end{array}$ & $\begin{array}{l}\text { Desig- } \\
\text { nated as }\end{array}$ & Colony characters & $\begin{array}{l}\text { Colony } \\
\text { diameter } \\
(\mathrm{mm})\end{array}$ & Pigmentation \\
\hline 25 & FS-25 & $\begin{array}{l}\text { white submerged and reddish } \\
\text { growth on the back side of the } \\
\text { petriplate }\end{array}$ & 51.33 & $\begin{array}{l}\text { Reddish } \\
\text { brown }\end{array}$ \\
\hline 26 & FS-26 & Cottony white submerged growth & 65.78 & Milky white \\
\hline 27 & FS-27 & Sumerged white fussy growth & 58.66 & Whitish \\
\hline 28 & FS-28 & Submerged dull white growth & 49.33 & Dull yellow \\
\hline 29 & FS-29 & $\begin{array}{l}\text { Cottony white raised medium } \\
\text { growth }\end{array}$ & 74.55 & - \\
\hline 30 & FS-30 & Fussy white raised growth & 72.44 & Light red \\
\hline 31 & FS-31 & $\begin{array}{l}\text { Reddish brown colour fussy and } \\
\text { fast growth }\end{array}$ & 82.00 & Brownish \\
\hline 32 & FS-32 & $\begin{array}{l}\text { Dull whitish yellow submerged but } \\
\text { raised at centre }\end{array}$ & 58.89 & Yellowish \\
\hline 33 & FS-33 & $\begin{array}{l}\text { Raised cottony white but lightly } \\
\text { yellowish at centre }\end{array}$ & 82.00 & - \\
\hline 34 & FS-34 & $\begin{array}{l}\text { Fussy dull white cottony raised } \\
\text { growth }\end{array}$ & 64.66 & - \\
\hline 35 & FS-35 & Whitist cottony raised growth & 83.11 & Dull white \\
\hline 36 & FS-36 & Dull white submerged growth & 82.44 & Dull white \\
\hline 37 & FS-37 & White yellowish fussy growth & 56.33 & Light red \\
\hline 38 & FS-38 & White fussy cottony growth & 71.11 & Dark brown \\
\hline 39 & FS-39 & Raised white fussy growth & 78.33 & Whitish \\
\hline 40 & FS-40 & $\begin{array}{l}\text { Yellowish white fussy red colour } \\
\text { developed at the back of petriplates }\end{array}$ & 81.66 & Light yellow \\
\hline 41 & FS-41 & White yellow submersed growth & 44.33 & - \\
\hline 42 & FS-42 & $\begin{array}{l}\text { Dull whitish to brown colour } \\
\text { medium raised growth }\end{array}$ & 71.11 & Yellowish \\
\hline 43 & FS-43 & Dull white creamy fast growth & 55.33 & Light red \\
\hline 44 & FS-44 & $\begin{array}{l}\text { Fussy white cottony submersed } \\
\text { growth, greenish colour appeared } \\
\text { in the middle }\end{array}$ & 71.11 & Light red \\
\hline 45 & FS-45 & Dull white submerged fast growth & 68.00 & Light red \\
\hline 46 & FS-46 & $\begin{array}{l}\text { Yellow white dull submersed } \\
\text { growth }\end{array}$ & 75.55 & Dark brown \\
\hline 47 & FS-47 & $\begin{array}{l}\text { Creamy white to yellowish } \\
\text { suppressed growth }\end{array}$ & 81.11 & Whitish \\
\hline 48 & FS-48 & Creamy white submerged growth & 75.00 & Dark brown \\
\hline 49 & FS-49 & Submerged dull white growth & 82.22 & Dark brown \\
\hline 50 & FS-50 & $\begin{array}{l}\text { Creamy white submerged fast } \\
\text { growth }\end{array}$ & 82.70 & Dark brown \\
\hline 51 & FS-51 & Dull white suppressed growth & 75.67 & Brownish \\
\hline 52 & FS-52 & White cottony submerged growth & 73.66 & Yellowish \\
\hline
\end{tabular}


Table-2: Categorization of Fusarium solani isolates on the basis of growth on PDA in $\mathrm{mm}$

\begin{tabular}{|c|c|l|c|}
\hline $\begin{array}{c}\text { Sr. } \\
\text { No. }\end{array}$ & $\begin{array}{c}\text { Growth } \\
\text { range }(\mathbf{m m})\end{array}$ & \multicolumn{1}{|c|}{ Isolates } & \multicolumn{1}{|c|}{$\begin{array}{c}\text { Total } \\
\text { Isolates }\end{array}$} \\
\hline 1 & $<50$ & FS-1, FS-6, FS-8, FS-41, FS-28. & 5 \\
\hline 2 & $51-60$ & $\begin{array}{l}\text { FS-25, FS-2, FS-43, FS-37, FS-27, FS-32, FS- } \\
4 .\end{array}$ & 7 \\
\hline 3 & $61-70$ & $\begin{array}{l}\text { FS-3, FS-34, FS-15, FS-16, FS-26, FS-45, FS- } \\
\mathbf{2 3}, \text { FS-7 }\end{array}$ & 8 \\
\hline 4 & $71-80$ & $\begin{array}{l}\text { FS-42, FS-38, FS-44, FS-24, FS-19, FS-30, } \\
\text { FS-10, FS-52, FS-20, FS-29, FS-48, FS-46, } \\
\text { FS-51, FS-5, FS-39, FS-22, FS-14 }\end{array}$ & 17 \\
\hline 5 & $>80$ & $\begin{array}{l}\text { FS-47, FS-21, FS-40, FS-18, FS-31, FS-33, } \\
\text { FS-49, FS-36, FS-50, FS-35, FS-12, FS-17, } \\
\text { FS-9, FS-13, FS-1 1. }\end{array}$ & 15 \\
\hline
\end{tabular}

